\title{
A characterisation of large finitely presented groups
}

\author{
Marc Lackenby \\ Mathematical Institute, Oxford University, 24-29 St Giles', Oxford OX1 3LB, UK \\ Received 25 March 2004 \\ Available online 5 April 2005 \\ Communicated by Alexander Lubotzky
}

\section{Introduction}

In this paper, we will consider finitely presented groups that have a finite index subgroup which admits a surjective homomorphism onto a non-abelian free group. Gromov called these groups large [4]. Large groups have particularly nice properties (for example, super-exponential subgroup growth). They also play an important rôle in low-dimensional topology: it is a major conjecture that the fundamental group of any closed hyperbolic 3-manifold is large. Our main theorem is a characterisation of these groups in terms of the existence of a normal series where successive quotients are finite abelian groups with sufficiently large rank and order.

Theorem 1.1. Let $G$ be a finitely presented group. Then the following are equivalent:

(1) some finite index subgroup of $G$ admits a surjective homomorphism onto a non-abelian free group;

(2) there exists a sequence $G_{1} \geqslant G_{2} \geqslant \cdots$ of finite index subgroups of $G$, each normal in $G_{1}$, such that

(i) $G_{i} / G_{i+1}$ is abelian for all $i \geqslant 1$;

(ii) $\lim _{i \rightarrow \infty}\left(\left(\log \left[G_{i}: G_{i+1}\right]\right) /\left[G: G_{i}\right]\right)=\infty$;

(iii) $\limsup _{i}\left(d\left(G_{i} / G_{i+1}\right) /\left[G: G_{i}\right]\right)>0$.

Here, $d($ ) denotes the rank of a group, which is its minimal number of generators. Note that condition (i) does not require $G / G_{1}$ to be an abelian group. Indeed, it can-

E-mail address: lackenby@maths.ox.ac.uk. 
not, since there are finitely presented groups that are both perfect and large. In words, condition (ii) requires the order of $G_{i} / G_{i+1}$ to grow super-exponentially as a function of [ $\left.G: G_{i}\right]$. Condition (iii) asserts that the rank of the quotients $G_{i} / G_{i+1}$ grows linearly in the index $\left[G: G_{i}\right]$. This is the fastest it could possibly grow, since the Reidermeister-Schreier theorem implies that the rank of $G_{i}$ grows at most linearly in $\left[G: G_{i}\right]$.

The main part of Theorem 1.1 , the implication $(2) \Rightarrow(1)$, is in fact a corollary of the following stronger, but slightly less elegant, result.

Theorem 1.2. Let $G$ be a finitely presented group, and suppose that, for each natural number $i$, there is a triple $H_{i} \geqslant J_{i} \geqslant K_{i}$ of finite index normal subgroups of $G$ such that

(i) $H_{i} / J_{i}$ is abelian for all $i$;

(ii) $\lim _{i \rightarrow \infty}\left(\left(\log \left[H_{i}: J_{i}\right]\right) /\left[G: H_{i}\right]\right)=\infty$;

(iii) $\lim \sup _{i}\left(d\left(J_{i} / K_{i}\right) /\left[G: J_{i}\right]\right)>0$.

Then $K_{i}$ admits a surjective homomorphism onto a free non-abelian group, for infinitely many $i$.

This gives $(2) \Rightarrow(1)$ of Theorem 1.1 , via the following argument. We may replace $G$ by $G_{1}$, and thereby assume that each $G_{i}$ is normal in $G$. If we then set $H_{i}=G_{i}, J_{i}=$ $G_{i+1}$ and $K_{i}=G_{i+2}$, Theorem 1.2 implies that infinitely many $G_{i}$ admit a surjective homomorphism onto a free non-abelian group.

In fact, the statements of Theorems 1.1 and 1.2 are not the strongest that can be made. The existence of infinitely many subgroups $G_{i}$ (in Theorem 1.1) or infinitely many triples $H_{i} \geqslant J_{i} \geqslant K_{i}$ (in Theorem 1.2) is more than one needs to deduce that $G$ is large. This conclusion still holds if one replaces hypotheses (ii) and (iii) of Theorem 1.2 by an explicit inequality which relates $\left[H_{i}: J_{i}\right],\left[G: H_{i}\right], d\left(J_{i} / K_{i}\right)$, for some fixed $i$, and data from a fixed presentation of $G$. The precise statement of this result, which is rather unwieldy, appears as Theorem 4.2 in Section 4.

What makes the results of this paper noteworthy is their method of proof. Despite the fact that these theorems are purely group-theoretic, their proof uses very little algebra. Instead, the geometry and topology of finite Cayley graphs play a central rôle. This is the second in a pair of papers that exploit these type of arguments. The first [5] related Property $(\tau)$, the rank of finite index subgroups and their possible decomposition as a graph of groups. Using this relationship, the proof of a weaker form of Theorem 1.1 was sketched.

Large groups were studied by Baumslag and Pride [1] who showed that groups with a presentation having (at least) two more generators than relations are of this form. This is an easy consequence of Theorem 1.1.

Corollary 1.3. Let $G$ be a group having a presentation with at least two more generators than relations. Then $G$ has a finite index subgroup that admits a surjective homomorphism onto a free non-abelian group. 
Baumslag and Pride [2] also conjectured that when a group $G$ has a presentation with one more generator than relation, but one of the relations is a proper power, then $G$ is large. This was proved independently by Gromov [4] using bounded cohomology and by Stöhr [10] using a direct algebraic argument. Again, this is a straightforward consequence of Theorem 1.1.

Corollary 1.4. Let $G$ be a group having a presentation with one more generator than relation. Suppose that one of these relations is proper power. Then $G$ has a finite index subgroup that admits a surjective homomorphism onto a free non-abelian group.

We will prove these corollaries in Section 5.

We will also show that conditions (i) and (ii) of Theorem 1.1 are, in fact, equivalent to the existence of a finite index subgroup with infinite abelianisation.

Theorem 1.5. Let $G$ be a finitely presented group. Then the following are equivalent:

(1) some finite index subgroup of $G$ has infinite abelianisation;

(2) there exists a sequence $G_{1} \geqslant G_{2} \geqslant \cdots$ of finite index subgroups of $G$, each normal in $G_{1}$, such that

(i) $G_{i} / G_{i+1}$ is abelian for all $i \geqslant 1$;

(ii) $\lim _{i \rightarrow \infty}\left(\left(\log \left[G_{i}: G_{i+1}\right]\right) /\left[G: G_{i}\right]\right)=\infty$.

The proof of this result, which is topological and rather straightforward, is given in Section 6.

\section{The forwards implication}

In this section, we will prove $(1) \Rightarrow(2)$ of Theorem 1.1 . Let $G_{1}$ be the finite index subgroup of $G$ that admits a surjective homomorphism onto a non-abelian free group $F$. Define recursively the following finite index normal subgroups of $F$. Set $L_{1}=F$, and, for $i \geqslant 1$, let $L_{i+1}=\left[L_{i}, L_{i}\right]\left(L_{i}\right)^{i}$. In other words, $L_{i+1}$ is the group generated by the commutators and the $i$ th powers of $L_{i}$. Thus, $\left\{L_{i}\right\}$ is a nested sequence of subgroups of $F$. Each is characteristic in the preceding one, and hence each is characteristic in $F$, and is therefore normal in $F$. Each $L_{i}$ is free with rank $d\left(L_{i}\right)=(d(F)-1)\left[F: L_{i}\right]+1$, and $L_{i} / L_{i+1}$ is isomorphic to $(\mathbb{Z} / i \mathbb{Z})^{d\left(L_{i}\right)}$.

Set $G_{i}$ to be the inverse image of $L_{i}$ in $G$, which is a normal subgroup of $G_{1}$. Then $G_{1} / G_{i}$ is isomorphic to $F / L_{i}$, and $G_{i} / G_{i+1}$ is isomorphic to $L_{i} / L_{i+1}$, which is abelian, verifying (i) of Theorem 1.1 . To check (iii), note that

$$
\begin{aligned}
d\left(G_{i} / G_{i+1}\right) & =d\left(L_{i} / L_{i+1}\right)=d\left(L_{i}\right)=(d(F)-1)\left[F: L_{i}\right]+1 \\
& =(d(F)-1)\left[G_{1}: G_{i}\right]+1
\end{aligned}
$$


Similarly, to establish (ii), we note that

$$
\log \left[G_{i}: G_{i+1}\right]=\log \left(i^{d\left(L_{i}\right)}\right)=d\left(L_{i}\right) \log i>\left[G_{1}: G_{i}\right] \log i .
$$

\section{The width and Cheeger constant of finite graphs}

Many of the ideas behind this paper arise from the theory of Property $(\tau)$. This is a particularly useful group-theoretic concept, introduced by Lubotzky and Zimmer [8], that can be defined using graph theory, representation theory or differential geometry [6]. We concentrate on the former approach. The Cheeger constant of a finite graph $X$, denoted $h(X)$, is defined to be

$$
\min \left\{\frac{|\partial A|}{|A|}: A \subset V(X) \text { and } 0<|A| \leqslant \frac{|V(X)|}{2}\right\} .
$$

Here, $V(X)$ denotes the vertex set of $X$, and, for a subset $A$ of $V(X), \partial A$ denotes the set of edges with one endpoint in $A$ and one not in $A$. Informally, having small Cheeger constant is equivalent to the existence of a 'bottleneck' in the graph. (See Fig. 1.)

Let $G$ be a group with a finite generating set $S$. Let $\left\{G_{i}\right\}$ be a collection of finite index normal subgroups. We denote the Cayley graph of $G / G_{i}$ with respect to $S$ by $X\left(G / G_{i} ; S\right)$. The group $G$ is said to have Property $(\tau)$ with respect to $\left\{G_{i}\right\}$ if the Cheeger constants $h\left(X\left(G / G_{i} ; S\right)\right)$ are bounded away from zero. This property turns out not to depend on the choice of finite generating set $S$.

Whether or not a given group and a collection of finite index subgroups have Property $(\tau)$ is a subtle and often difficult question. The following theorem of Lubotzky and Weiss [7] gives a necessary condition for a group to have Property $(\tau)$. This is not, in fact, how Lubotzky and Weiss stated their result (which appears as Theorem 3.6 of [7]), but this formulation can readily be deduced from their argument.

Theorem 3.1. Suppose that a finitely generated group $G$ has Property $(\tau)$ with respect to a collection $\mathcal{C}$ of finite index normal subgroups. Then there is a constant $c$ with the following property. If $J$ is a member of $\mathcal{C}$, and $J$ is contained in a normal subgroup $H$ of $G$ such that $H / J$ is abelian, then $|H / J|<c^{[G: H]}$.

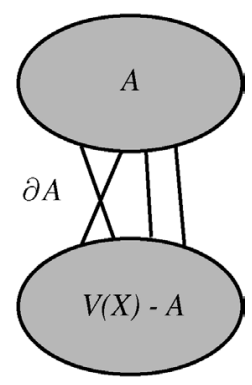

Fig. 1. 
Thus, conditions (i) and (ii) of Theorem 1.2 imply that $G$ does not have Property $(\tau)$ with respect to $\left\{J_{i}\right\}$. We will actually need to establish a stronger version of Theorem 3.1. Instead of relating to the Cheeger constant of $X\left(G / J_{i} ; S\right)$ (for some finite generating set $S$ ), we need to consider a related geometric invariant of $X\left(G / J_{i} ; S\right)$, its width, which is defined as follows.

Let $X$ be a finite graph. Consider a linear ordering on its vertices. For $0 \leqslant n \leqslant|V(X)|$, let $D_{n}$ be the first $n$ vertices. The width of the ordering is defined to be $\max _{n}\left|\partial D_{n}\right|$. The width of the graph is the minimal width of any of its orderings, and is denoted $w(X)$.

This notion is inspired by a useful concept from the theory of knots and 3-manifolds, known as thin position [9], which was first introduced by Gabai [3]. We now develop this analogy (which is not essential for an understanding of the remainder of the paper). One may imagine the graph $X$ embedded in $\mathbb{R}^{3}$, with its vertices all at distinct heights, and with its edges realised as straight lines. The height of the vertices specifies a linear ordering on them. The width of this ordering can be interpreted geometrically, as follows. Imagine a 1-parameter family of horizontal planes, parametrised by their heights which increase monotonically from $-\infty$ to $\infty$. The width of the ordering is equal to the maximal number of intersections between the graph and any of these planes. (See Fig. 2 for an example.) Thus, to determine the width of $X$, one should aim to find the most efficient embedding in $\mathbb{R}^{3}$ : the one that minimises the width of the associated ordering. This is highly analogous to thin position for knots in $\mathbb{R}^{3}$, where one aims to isotope the knot until a similar notion of width is minimised.

There is a relationship between the width of a graph and its Cheeger constant. In an ordering on $V(X)$ of minimal width, consider $D_{n}$ where $n=\lfloor|V(X)| / 2\rfloor$. By computing $\left|\partial D_{n}\right| /\left|D_{n}\right|$, we deduce that

$$
h(X) \leqslant \frac{w(X)}{\lfloor|V(X)| / 2\rfloor} .
$$

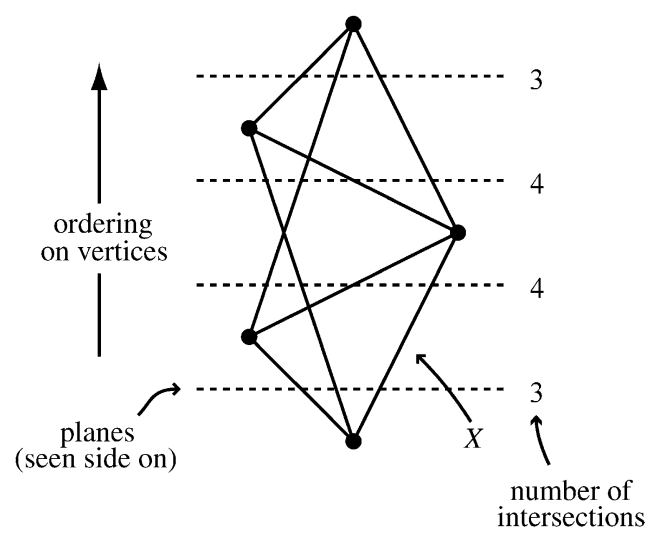

Fig. 2. 
Hence, the following gives a stronger version of Theorem 3.1. It gives a condition guaranteeing that certain Cayley graphs have width which is asymptotically smaller than their number of vertices.

Theorem 3.2. Let $G$ be a group with a finite generating set $S$. Suppose that, for each natural number $i$, there is a pair $H_{i} \geqslant J_{i}$ of finite index normal subgroups of $G$, such that

(i) $H_{i} / J_{i}$ is abelian for all $i$;

(ii) $\lim _{i \rightarrow \infty}\left(\left(\log \left[H_{i}: J_{i}\right]\right) /\left[G: H_{i}\right]\right)=\infty$.

Then $w\left(X\left(G / J_{i} ; S\right)\right) /\left[G: J_{i}\right] \rightarrow 0$.

Note that (i) and (ii) are precisely those in Theorem 1.2.

The proof we give of this result follows the argument of Lubotzky and Weiss in their proof of Theorem 3.1. The following lemma allows us to estimate the width of $X\left(G / J_{i} ; S\right)$ in terms of the width of a Cayley graph of $H_{i} / J_{i}$. This will be useful, since $H_{i} / J_{i}$ is abelian, and, later, we will analyse the width of Cayley graphs of finite abelian groups.

Lemma 3.3. Let $G$ be a group with a finite generating set $S$, and let $H_{i} \geqslant J_{i}$ be finite index normal subgroups of $G$. Let $\Sigma$ be a generating set for $H_{i}$ arising from $S$ by the Reidermeister-Schreier process. Then

$$
w\left(X\left(G / J_{i} ; S\right)\right) \leqslant w\left(X\left(H_{i} / J_{i} ; \Sigma\right)\right)+2|S|\left[G: H_{i}\right] .
$$

Proof. We first recall the Reidermeister-Schreier process. Pick a presentation for $G$ with generating set $S$, but possibly an infinite number of relations. (We are not assuming here that $G$ is finitely presented.) Build the associated 2-complex $C$, by starting with a bouquet of $|S|$ circles and attaching on a 2-cell for each relation. Then $\pi_{1}(C)$ is isomorphic to $G$. Let $C^{\prime} \rightarrow C$ be the covering corresponding to the subgroup $H_{i}$, so that $\pi_{1}\left(C^{\prime}\right)$ is isomorphic to $H_{i}$. Pick a maximal tree $T$ in the 1-skeleton of $C^{\prime}$. Collapsing this tree to a point gives a new 2-complex $\bar{C}^{\prime}$. Its 1-cells give the required generating set $\Sigma$ for $H_{i}$.

Let $C^{\prime \prime} \rightarrow C^{\prime}$ and $\bar{C}^{\prime \prime} \rightarrow \bar{C}^{\prime}$ be the coverings corresponding to $J_{i}$. The inverse image of $T$ in $C^{\prime \prime}$ is a forest $F$. If one were to collapse each component of this forest to a single vertex, we would obtain $\bar{C}^{\prime \prime}$. The 1 -skeletons of $C^{\prime \prime}$ and $\bar{C}^{\prime \prime}$ are, respectively, $X\left(G / J_{i} ; S\right)$ and $X\left(H_{i} / J_{i} ; \Sigma\right)$. (See Fig. 3.)

Consider a minimal width ordering of $V\left(\bar{C}^{\prime \prime}\right)$. From this, one can construct an ordering of $V\left(C^{\prime \prime}\right)$, as follows. The ordering on $V\left(\bar{C}^{\prime \prime}\right)$ specifies an ordering on the components of $F$. We therefore order $V\left(C^{\prime \prime}\right)$ by ordering the vertices of its first tree in some way, then the vertices of its second tree, and so on.

For any integer $n$, with $0 \leqslant n \leqslant\left|V\left(C^{\prime \prime}\right)\right|$, let $D_{n}$ be the first $n$ vertices of $C^{\prime \prime}$. Suppose that the $n$th vertex of $C^{\prime \prime}$ lies in a component $\widetilde{T}$ of $F$. Then, the edges in $\partial D_{n}$ consist of edges joining trees other than $\widetilde{T}$, and edges with at least one endpoint in $\widetilde{T}$. There are at most $w\left(X\left(H_{i} / J_{i} ; \Sigma\right)\right)$ edges of the first type and at most $2|S|\left[G: H_{i}\right]$ edges of the second type. Thus the width of $C^{\prime \prime}$ is at most the sum of these quantities. 

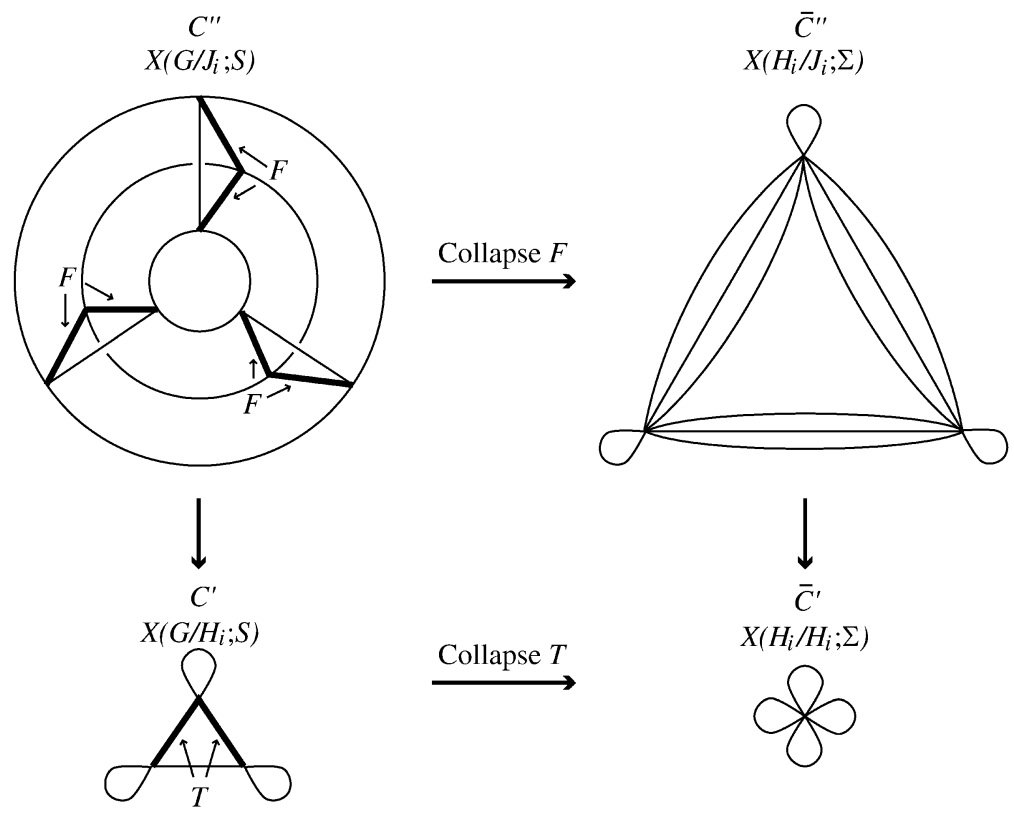

Fig. 3.

As a result of the above lemma, we concentrate on the Cayley graph of the abelian group $H_{i} / J_{i}$. The following lemma provides a useful upper bound on its width.

Lemma 3.4. Let A be a finite abelian group with finite generating set $\Sigma$. Then

$$
w(X(A ; \Sigma)) \leqslant \frac{6|\Sigma||A|}{\left\lfloor(|A|-1)^{1 /|\Sigma|\rfloor}\right.} .
$$

Proof. We will construct an efficient ordering of the vertices of $X(A ; \Sigma)$ by placing them on the unit circle in a suitable way. Give the circle a group structure, by identifying it with $\mathbb{C}^{\times}$, the multiplicative group of complex numbers with modulus one. Any homomorphism $\phi: A \rightarrow \mathbb{C}^{\times}$determines a point in $\left(\mathbb{C}^{\times}\right)^{|\Sigma|}$, given by the $|\Sigma|$-tuple $(\phi(s): s \in \Sigma)$. Define $c$ to be $\left\lfloor(|A|-1)^{1 /|\Sigma|}\right\rfloor$, which is the denominator in the upper bound on $w(X(A ; \Sigma))$ that we are trying to establish. We may assume that $c$ is a positive integer; otherwise, there is nothing to prove. Divide the circle $\mathbb{C}^{\times}$into $c$ equal arcs. This determines a decomposition of $\left(\mathbb{C}^{\times}\right)^{|\Sigma|}$ into $c^{|\Sigma|}<|A|$ boxes. There are precisely $|A|$ distinct homomorphisms $A \rightarrow \mathbb{C}^{\times}$, and hence two distinct homomorphisms are sent to the same box. Their quotient is a non-trivial homomorphism $\phi: A \rightarrow \mathbb{C}^{\times}$, such that $|\arg (\phi(s))| \leqslant 2 \pi / c$ for all $s \in \Sigma$. Here, we are taking arguments to lie in the range $(-\pi, \pi]$.

Let $\sigma$ be the element of $A / \operatorname{Ker}(\phi)$, such that $\phi(\sigma)$ has smallest positive argument. Then $\sigma$ is a generator for $A / \operatorname{Ker}(\phi)$. Let $N$ be its order. Note that, for any $s \in \Sigma$ that does not lie in $\operatorname{Ker}(\phi), N \geqslant 2 \pi /|\arg (\phi(s))| \geqslant c$. Since this is true for at least one $s \in \Sigma$, we deduce that $N \geqslant c$. 


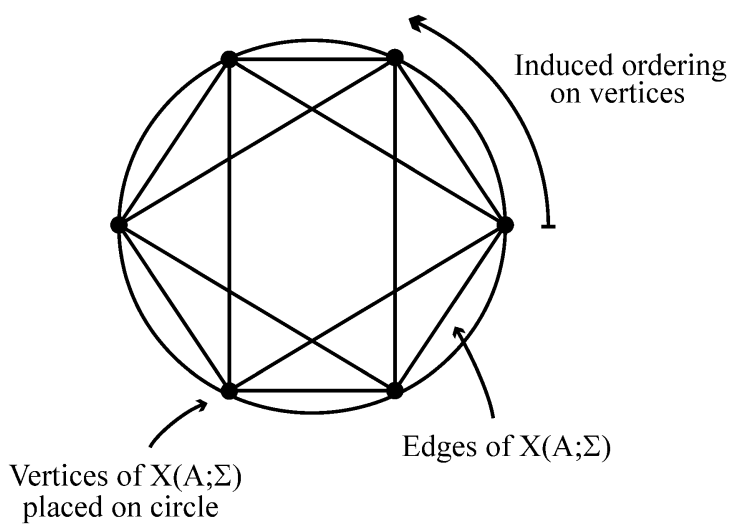

Fig. 4.

We place an order on the vertices of $A$, as follows. First order the vertices in $\operatorname{Ker}(\phi)$ in some way, then order the coset $\phi^{-1}(\sigma)$, then $\phi^{-1}\left(\sigma^{2}\right)$, and so on. (See Fig. 4.)

Note that any edge in $X(A ; \Sigma)$ joins vertices who images in $A / \operatorname{Ker}(\phi)$ differ by $\sigma^{i}$, where $|i|$ is at most $N / c$. Let $D_{n}$ be a subset of the vertices of $X(A ; \Sigma)$, as in the definition of width. If we consider the initial vertex of an edge in $\partial D_{n}$, its image in $A / \operatorname{Ker}(\phi)$ can take at most $4(N / c)+2$ possible values. This is at most $6(N / c)$, since $N / c \geqslant 1$. Hence, there are at most $6(N / c)|\operatorname{Ker}(\phi)|=6|A| / c$ such vertices. Since $|\Sigma|$ oriented edges emanate from any vertex, there are therefore at most $6|\Sigma||A| / c$ edges in $\partial D_{n}$. This gives the required upper bound on the width of $X(A ; \Sigma)$.

Proof of Theorem 3.2. By Lemma 3.3,

$$
\frac{w\left(X\left(G / J_{i} ; S\right)\right)}{\left[G: J_{i}\right]} \leqslant \frac{w\left(X\left(H_{i} / J_{i} ; \Sigma\right)\right)}{\left[G: J_{i}\right]}+\frac{2|S|\left[G: H_{i}\right]}{\left[G: J_{i}\right]} .
$$

By assumption (ii), [ $\left.H_{i}: J_{i}\right]$ grows super-exponentially as a function of $\left[G: H_{i}\right]$. In particular, $\left[H_{i}: J_{i}\right]$ tends to infinity, and so the second term tends to zero. For the first term, assumption (i) and Lemma 3.4 imply that

$$
\frac{w\left(X\left(H_{i} / J_{i} ; \Sigma\right)\right)}{\left[G: J_{i}\right]} \leqslant \frac{6|\Sigma|}{\left[G: H_{i}\right]\left\lfloor\left(\left[H_{i}: J_{i}\right]-1\right)^{1 /|\Sigma|}\right\rfloor} .
$$

From the Reidermeister-Schreier process, $|\Sigma|$ grows linearly in $\left[G: H_{i}\right]$. So, $|\Sigma| /\left[G: H_{i}\right]$ is bounded above and, by assumption (ii), $\left(\left[H_{i}: J_{i}\right]-1\right)^{1 /|\Sigma|}$ tends to infinity.

\section{Proof of the main theorem}

Theorem 1.2 follows immediately from Theorem 3.2 and the following result. 
Theorem 4.1. Let $G$ be a finitely presented group with a finite generating set $S$. Suppose that, for each natural number $i$, there is a pair $J_{i} \geqslant K_{i}$ of finite index normal subgroups of $G$, such that

(I) $w\left(X\left(G / J_{i} ; S\right)\right) /\left[G: J_{i}\right] \rightarrow 0$;

(II) $\lim \sup _{i}\left(d\left(J_{i} / K_{i}\right) /\left[G: J_{i}\right]\right)>0$.

Then, for infinitely many $i, K_{i}$ admits a surjective homomorphism onto a free non-abelian group.

Note that in the above theorem, we no longer need the hypothesis that any quotient groups are abelian.

The proof we give of Theorem 4.1 resembles some of the arguments in [5]. Suppose that (I) and (II) of the theorem hold. Let $C$ be a finite 2-complex having fundamental group $G$, arising from a finite presentation of $G$ with generating set $S$. Thus, $C$ has a single vertex, and an oriented edge for each element of $S$. Let $L$ be the sum of the lengths of the relations in this presentation, which we may assume is at least one.

Let $C_{i} \rightarrow C$ be the covering corresponding to $J_{i}$, and let $X_{i}=X\left(G / J_{i} ; S\right)$ be the 1-skeleton of $C_{i}$. Set $\ell$ to be

$$
\underset{i}{\limsup } \frac{d\left(J_{i} / K_{i}\right)}{\left[G: J_{i}\right]},
$$

which by (II) is positive. For infinitely many $i$,

$$
\frac{d\left(J_{i} / K_{i}\right)}{\left[G: J_{i}\right]}>\frac{3 \ell}{4} .
$$

By (I), $w\left(X_{i}\right) /\left[G: J_{i}\right] \rightarrow 0$. Hence, provided $i$ is sufficiently large,

$$
\frac{w\left(X_{i}\right)}{\left[G: J_{i}\right]}<\frac{\ell}{8 L} .
$$

Also, for all large $i$,

$$
\left[G: J_{i}\right] \geqslant \frac{8\left(2|S|+L^{2}\right)}{\ell} .
$$

We now fix some $i$ so that the inequalities (1), (2) and (3) hold.

We will show that there is a surjective homomorphism from $K_{i}$ onto a free non-abelian group. The details of this argument are a little complicated, but the main idea is fairly simple. We use a minimal width ordering on the vertices of $X_{i}$ to divide $C_{i}$ into two pieces $A$ and $B$ that are, roughly, equally 'big.' More precisely, the images of their fundamental groups in $J_{i} / K_{i}$ both have large rank. (In fact, $A$ and $B$ might not be connected, and so it might not make sense to refer to their fundamental group, but we will ignore this point for the moment.) Since the width of $X_{i}$ is small, the fundamental group of $A \cap B$ has small 
rank, as therefore does its image in $J_{i} / K_{i}$. So, when the decomposition of $C_{i}$ into $A$ and $B$ is lifted to the covering space corresponding to $K_{i}$, the lifted decomposition is modelled on a graph with negative Euler characteristic. The desired conclusion, that $K_{i}$ surjects onto a non-abelian free group, follows immediately.

We now give this argument in detail. Pick a minimal width ordering on the vertices of $X_{i}$. We will use this to define a height function $f: C_{i} \rightarrow \mathbb{R}$. On the vertices, simply let $f$ agree with the ordering function. Extend linearly over the 1-cells of $C_{i}$. Then we extend $f$ continuously over the interior of each 2-cell, so that it is a Morse function there, with the following properties. (There is one exceptional case: when all the vertices in the boundary of a 2-cell are in fact the same 0-cell in $C_{i}$ and hence have the same height, then we define $f$ to be constant on that 2-cell.) We can ensure that $f$ has no maxima or minima in the interior of any 2-cell, and that its critical values avoid $n+1 / 2$, for each integer $n$. We can also ensure that, whenever a 2-cell does not contains the vertex of height $n$ in its boundary, then that 2-cell contains no critical points with values between $n-1 / 2$ and $n+1 / 2$.

Let $n$ be some integer between 0 and $\left|V\left(X_{i}\right)\right|$. We will focus on a single value of $n$ later. We can now use $n$ and $f$ to decompose $C_{i}$ into two subsets $A_{n}=f^{-1}(-\infty, n+1 / 2]$ and $B_{n}=f^{-1}[n+1 / 2, \infty)$. Let $D_{n}$ be the vertices of $C_{i}$ that lie in $A_{n}$; these are precisely the first $n$ vertices of the ordering.

Note that $A_{n} \cap B_{n}$ is a 1-complex, with precisely one 0 -cell in the interior of each edge of $\partial D_{n}$, and no other 0-cells. Hence, there are exactly $\left|\partial D_{n}\right| 0$-cells of $A_{n} \cap B_{n}$. This therefore is an upper bound on the number of components of $A_{n} \cap B_{n}$, and hence on the number of components of $A_{n}$ and on the number of components of $B_{n}$ (provided $\left.n \neq 0,\left|V\left(X_{i}\right)\right|\right)$. Note that $\left|\partial D_{n}\right| \leqslant w\left(X_{i}\right)<\frac{1}{8} \ell\left[G: J_{i}\right]$, by inequality (2).

The 1-cells of $A_{n} \cap B_{n}$ are arcs properly embedded in the 2-cells of $C_{i}$. We claim that $A_{n} \cap B_{n}$ has at most $\left|\partial D_{n}\right| L / 21$-cells. Note that the total number of times the 2-cells of $C_{i}$ run over any 1-cell of $C_{i}$ is at most $L$. This is an upper bound for the valence of each 0-cell of $A_{n} \cap B_{n}$. There are precisely $\left|\partial D_{n}\right| 0$-cells of $A_{n} \cap B_{n}$, and hence we obtain the required bound on the number of 1-cells of $A_{n} \cap B_{n}$. Note that this bound $\left|\partial D_{n}\right| L / 2$ is at most $w\left(X_{i}\right) L / 2$, which is less than $\frac{1}{8} \ell\left[G: J_{i}\right]$, by inequality (2).

We construct a graph $Y_{n}$, as follows. Each vertex corresponds to a component of $A_{n}$ or $B_{n}$, and is labelled $A_{n}$ or $B_{n}$ as appropriate. Each edge corresponds to a component of $A_{n} \cap B_{n}$. Incidence in the graph is defined by topological incidence. (See Fig. 5.) Since $A_{n} \cap B_{n}$ has a small regular neighbourhood that is homeomorphic to $\left(A_{n} \cap B_{n}\right) \times I$, we may define a collapsing map $C_{i} \rightarrow Y_{n}$ that sends each component of $C_{i}-\left(\left(A_{n} \cap B_{n}\right) \times I\right)$ to the corresponding vertex of $Y_{n}$, and that sends each component of $\left(A_{n} \cap B_{n}\right) \times I$ to the relevant edge of $Y_{n}$, via projection onto the second factor of the product. However, $Y_{n}$ need not be very interesting: it may only be a single edge, for example. But a similar construction in the covering space of $C_{i}$ corresponding to the subgroup $K_{i}$ will induce the required surjective homomorphism from $K_{i}$ onto a free non-abelian group.

Define the weight wt ( ) of a vertex or edge, corresponding to a component $E$ of $A_{n}, B_{n}$ or $A_{n} \cap B_{n}$, to be $d\left(i_{*} \pi_{1} E / i_{*} \pi_{1} E \cap K_{i}\right)$, where $i_{*}: \pi_{1} E \rightarrow \pi_{1} C_{i}$ is the homomorphism induced by inclusion. (Note that the choice of basepoints for $\pi_{1} E$ does not affect this quantity.) Define the weight wt ( ) of $A_{n}, B_{n}$ or $A_{n} \cap B_{n}$ to be the sum of the weights of its components. Note that $w t\left(A_{n} \cap B_{n}\right)$ is at most the number of 1-cells of $A_{n} \cap B_{n}$, which we have already established is less than $\frac{1}{8} \ell\left[G: J_{i}\right]$. 

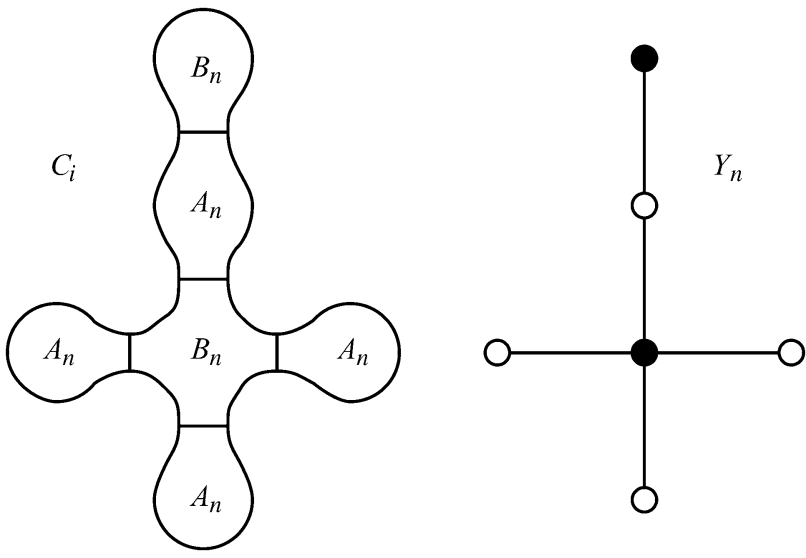

Fig. 5.

Claim. There is some $n$ such that $w t\left(A_{n}\right)$ and $w t\left(B_{n}\right)$ are each at least $\frac{1}{4} \ell\left[G: J_{i}\right]$.

Define

$$
\begin{aligned}
& Q=\left\{n: w t\left(A_{n}\right)<\frac{1}{4} \ell\left[G: J_{i}\right]\right\}, \\
& R=\left\{n: w t\left(B_{n}\right)<\frac{1}{4} \ell\left[G: J_{i}\right]\right\} .
\end{aligned}
$$

The aim is to show that $Q$ and $R$ do not cover the interval from 0 to $\left|V\left(X_{i}\right)\right|$. Since $J_{i}$ is generated by the elements of $i_{*} \pi_{1} E$, where $E$ runs over the components of $A_{n}$ and $B_{n}$, together with at most one generator for each component of $A_{n} \cap B_{n}$, we have the inequality

$$
d\left(J_{i} / K_{i}\right) \leqslant w t\left(A_{n}\right)+w t\left(B_{n}\right)+\left|A_{n} \cap B_{n}\right|,
$$

and so, by the above bound on $\left|A_{n} \cap B_{n}\right|$ and inequality (1),

$$
w t\left(A_{n}\right)+w t\left(B_{n}\right) \geqslant d\left(J_{i} / K_{i}\right)-\frac{1}{8} \ell\left[G: J_{i}\right]>\frac{5}{8} \ell\left[G: J_{i}\right] .
$$

Hence, when $n \in Q, w t\left(B_{n}\right)>\frac{3}{8} \ell\left[G: J_{i}\right]$ and, when $n \in R, w t\left(A_{n}\right)>\frac{3}{8} \ell\left[G: J_{i}\right]$. Thus, $Q$ and $R$ are disjoint. Note that $0 \in Q$ and $\left|V\left(X_{i}\right)\right| \in R$. Hence, the only way $Q$ and $R$ could cover the interval from 0 to $\left|V\left(X_{i}\right)\right|$ is if $n \in Q$ and $n+1 \in R$ for some $n$. This implies that

$$
w t\left(A_{n}\right)<\frac{1}{4} \ell\left[G: J_{i}\right], \quad w t\left(A_{n+1}\right)>\frac{3}{8} \ell\left[G: J_{i}\right]
$$

However, we shall now show that $w t\left(A_{n}\right)$ and $w t\left(A_{n+1}\right)$ differ by at most $2|S|+L^{2}$. Since $2|S|+L^{2} \leqslant \frac{1}{8} \ell\left[G: J_{i}\right]$, by inequality (3), this will provide a contradiction. It is clear that $A_{n+1}$ contains $A_{n}$. Only one vertex of $C_{i}$ lies in $A_{n+1}$ but not $A_{n}$; this is the $(n+1)$-st vertex of the ordering, called $x$, say. In the 1-cells and 2-cells of $C_{i}$ that are disjoint from $x$, $A_{n}$ and $A_{n+1}$ differ only by a small collar. Hence, we need only focus on the 1-cells and 
2-cells that are adjacent to $x$. There are at most $2|S|$ of these 1-cells, and at most $L$ 2-cells. In each 2-cell adjacent to $x$, we can obtain $A_{n+1}$ from $A_{n}$ by adding on a collection of discs that intersect $A_{n}$ and the boundary of the 2-cell in a total of at most $L$ arcs. Hence, it is clear that the weights of $A_{n+1}$ and $A_{n}$ differ by at most $2|S|+L^{2}$. This proves the claim.

We now fix $n$ as in the claim, and abbreviate $A_{n}, B_{n}, A_{n} \cap B_{n}$ and $Y_{n}$ to $A, B, A \cap B$ and $Y$. For any vertex $u$ of $Y$, let $g(u)$ denote its weight minus the total weight of the edges to which it is incident. Then the sum of $g(u)$, over all vertices $u$ of $Y$ labelled $A$, is $w t(A)-w t(A \cap B)$, which is more than $\frac{1}{8} \ell\left[G: J_{i}\right]$, and this is more than the number of vertices labelled $A$. Hence, there is some vertex $u$ labelled $A$, with $g(u)>1$. Similarly, there is some vertex $v$ labelled $B$ with $g(v)>1$. Let $P$ be an embedded path in $Y$ from $u$ to $v$.

Let $p: \widetilde{C}_{i} \rightarrow C_{i}$ be the covering corresponding to the subgroup $K_{i}$. The decomposition of $C_{i}$ into $A$ and $B$ pulls back to form a similar decomposition of $\widetilde{C}_{i}$. We obtain a similar graph $\widetilde{Y}$. The covering map $p: \widetilde{C}_{i} \rightarrow C_{i}$ induces a map of graphs $\widetilde{Y} \rightarrow Y$. (See Fig. 6.) Let $\widetilde{P}$ be the inverse image of $P$ in $\widetilde{Y}$.

The valence of each vertex of $\widetilde{P}$ is at least that of its image in $P$. When this image is not an endpoint of $P$, the valence is therefore at least two. We shall show that each inverse image of $u$ and $v$ has at least three edges of $\widetilde{P}$ emanating from it. Let $e$ be the edge of $P$ incident to $u$. Let $U$ and $E$ be the components of $A$ and $A \cap B$ corresponding to $u$ and $e$.

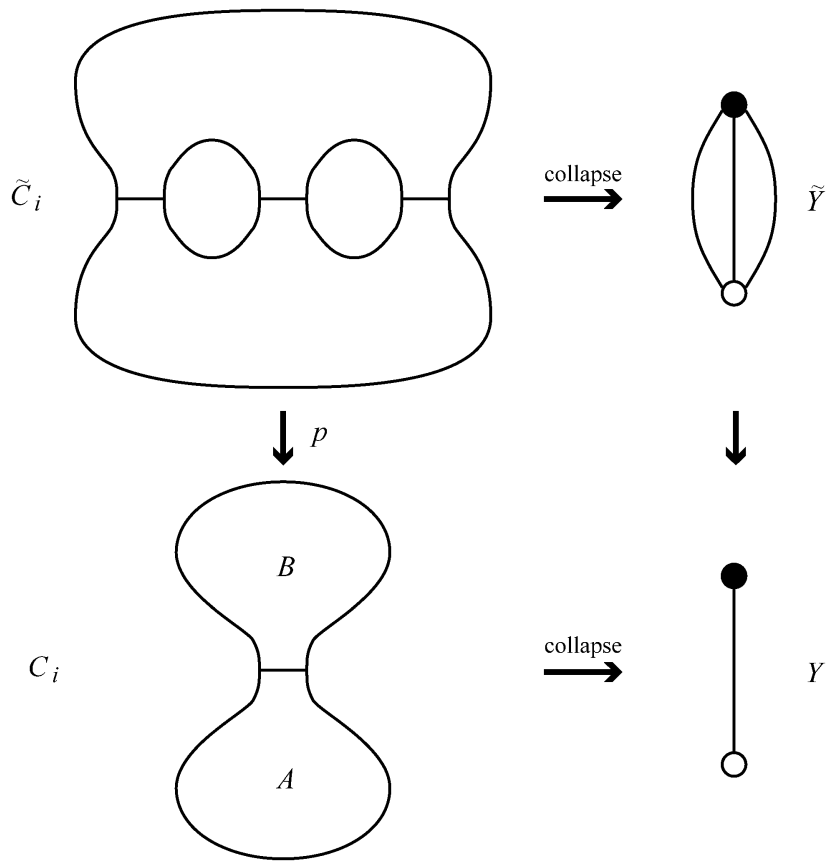

Fig. 6 . 
Claim. For any vertex in $\widetilde{P}$ that maps to $u$, the number of edges of $\widetilde{P}$ to which it is incident is

$$
\frac{\left[i_{*} \pi_{1} U: i_{*} \pi_{1} U \cap K_{i}\right]}{\left[i_{*} \pi_{1} E: i_{*} \pi_{1} E \cap K_{i}\right]} .
$$

Since $p: \widetilde{C}_{i} \rightarrow C_{i}$ is a regular cover, it has a group of covering transformations. This descends to a group action on $\widetilde{Y}$ which acts transitively on the vertices that map to $u$ and acts transitively on the edges that map to $e$. Hence, we need only show that, in $\widetilde{Y}$, the number of $e$-labelled edges and the number of $u$-labelled vertices are in the ratio given by the above formula. In fact, we will show that the number of these edges and vertices are, respectively,

$$
\frac{\left[J_{i}: K_{i}\right]}{\left[i_{*} \pi_{1} E: i_{*} \pi_{1} E \cap K_{i}\right]}, \quad \frac{\left[J_{i}: K_{i}\right]}{\left[i_{*} \pi_{1} U: i_{*} \pi_{1} U \cap K_{i}\right]},
$$

which will prove the claim. Consider one such edge. It corresponds to a component $Z$ of $p^{-1}(E)$. Now, $\left.p\right|_{Z}: Z \rightarrow E$ is a covering map. It corresponds to the subgroup of $\pi_{1}(E)$ consisting of based loops in $E$ that lift to loops in $\widetilde{C}_{i}$. This subgroup is $i_{*}^{-1} K_{i}$, and hence the degree of $\left.p\right|_{Z}$ is $\left[\pi_{1} E: i_{*}^{-1} K_{i}\right]=\left[i_{*} \pi_{1} E: i_{*} \pi_{1} E \cap K_{i}\right]$. But the degree of $p$ is $\left[J_{i}: K_{i}\right]$, and so the number of covering translates of $Z$ in $\widetilde{C}_{i}$, which is the number of $e$-labelled edges, is given by the required formula. The same argument applies to the $u$-labelled vertices, proving the claim.

Now,

$$
d\left(i_{*} \pi_{1} U / i_{*} \pi_{1} U \cap K_{i}\right)-d\left(i_{*} \pi_{1} E / i_{*} \pi_{1} E \cap K_{i}\right)=w t(u)-w t(e) \geqslant g(u)>1 .
$$

Hence $i_{*} \pi_{1} E / i_{*} \pi_{1} E \cap K_{i}$ has index more than two in $i_{*} \pi_{1} U / i_{*} \pi_{1} U \cap K_{i}$. Thus, the valence of any $u$-labelled vertex in $\widetilde{P}$ is more than two. The same argument applies to $v$. Hence, every edge of $\widetilde{P}$ has valence at least two, and at least two vertices have valence at least three. Therefore, $\chi(\widetilde{P})<0$, and so $\chi(\widetilde{Y})<0$. Thus, $\pi_{1}(\widetilde{Y})$ is a free non-abelian group. But the collapsing map $\widetilde{C}_{i} \rightarrow \widetilde{Y}$ induces a surjective homomorphism $K_{i} \rightarrow \pi_{1}(\widetilde{Y})$. This proves Theorem 4.1 and hence Theorem 1.2.

Theorem 1.2 can be strengthened to give the following, rather technical result. Unlike Theorem 1.2, this makes a hypothesis about just one triple $H \geqslant J \geqslant K$ of finite index normal subgroups of $G$.

Theorem 4.2. Let $G$ be a group with finite presentation $\langle S \mid R\rangle$. Let $L$ be the sum of the lengths of the relations $R$, which we assume is at least one. Suppose that $G$ has finite index normal subgroups $H \geqslant J \geqslant K$ such that $H / J$ is abelian and

$$
d(J / K)>\max \left\{\frac{48 L|S|}{\left\lfloor([H: J]-1)^{\left.[G: H]^{-1}|S|^{-1}\right\rfloor}\right.}+\frac{16 L|S|}{[H: J]}, 16|S|+8 L^{2}\right\} .
$$

Then $K$ admits a surjective homomorphism onto a non-abelian free group. 
Proof. All that one needed to make the proof of Theorem 4.1 work was the existence of a positive real number $\ell$ such that inequalities (1), (2) and (3) all hold. If we set $\ell$ to be $d(J / K) /[G: J]$, then (1) automatically is satisfied, and so we have reduced this to two inequalities. Inequality (3) becomes $d(J / K) \geqslant 16|S|+8 L^{2}$, which is part of our hypothesis. To guarantee that inequality (2) holds, we use the inequalities in the proof of Theorem 3.2 (where $\Sigma$ is as defined in Lemma 3.3):

$$
\begin{aligned}
\frac{w(X(G / J ; S))}{[G: J]} & \leqslant \frac{w(X(H / J ; \Sigma))}{[G: J]}+\frac{2|S|[G: H]}{[G: J]} \\
& \leqslant \frac{6|\Sigma|}{[G: H]\left\lfloor([H: J]-1)^{\left.|\Sigma|^{-1}\right\rfloor}+\frac{2|S|}{[H: J]}\right.} \\
& \leqslant \frac{6|S|[G: H]}{[G: H]\left\lfloor([H: J]-1)^{\left.[G: H]^{-1}|S|^{-1}\right\rfloor}\right.}+\frac{2|S|}{[H: J]} \\
& <\frac{d(J / K)}{8 L},
\end{aligned}
$$

by our hypothesis. Hence, the proof of Theorem 4.1 gives a surjective homomorphism from $K$ onto a free non-abelian group.

\section{Groups with more generators than relations}

In this section, we prove Corollaries 1.3 and 1.4.

Proof of Corollary 1.3. Let $\langle X \mid R\rangle$ be a presentation for $G$ with $|X|-|R|>1$. We define a nested sequence of finite index normal subgroups $G=G_{1} \geqslant G_{2} \geqslant \cdots$ recursively, by setting $G_{i+1}=\left[G_{i}, G_{i}\right]\left(G_{i}\right)^{i}$. Note that each is a characteristic subgroup of its predecessor, and so they are all normal in $G$.

The Reidermeister-Schreier process provides a presentation for $G_{i}$ with a total of $(|X|-1)\left[G: G_{i}\right]+1$ generators and $|R|\left[G: G_{i}\right]$ relations. Hence, the abelianisation of $G_{i}$ contains $\mathbb{Z}^{c_{i}}$ as a summand, where $c_{i}=(|X|-1-|R|)\left[G: G_{i}\right]+1$. Therefore, $G_{i} / G_{i+1}$ is an abelian group with $(\mathbb{Z} / i \mathbb{Z})^{c_{i}}$ as a summand. So, (i) of Theorem 1.1 holds. We now verify (ii) and (iii):

$$
d\left(G_{i} / G_{i+1}\right) \geqslant c_{i}=(|X|-1-|R|)\left[G: G_{i}\right]+1,
$$

which gives (iii), and

$$
\log \left[G_{i}: G_{i+1}\right] \geqslant \log \left(i^{c_{i}}\right)=\left((|X|-1-|R|)\left[G: G_{i}\right]+1\right) \log i
$$

which implies (ii). So, Theorem 1.1 now gives the corollary.

Proof of Corollary 1.4. This is similar to the above proof. Again, let $\langle X \mid R\rangle$ be the finite presentation of $G$, with $|X|-|R|=1$. Let $w^{q}$ be the relation in $R$ that is a proper 
power. We may assume that $q$ is prime. Define a nested sequence of finite index normal subgroups $G=G_{1} \geqslant G_{2} \geqslant \cdots$ recursively, by setting $G_{i+1}=\left[G_{i}, G_{i}\right]\left(G_{i}\right)^{p_{i}}$, where $p_{i}$ is the $i$ th prime bigger than $q$. Again, the Reidermeister-Schreier process gives a presentation for $G_{i}$ with $(|X|-1)\left[G: G_{i}\right]+1$ generators and $|R|\left[G: G_{i}\right]$ relations. This has one more generator that relation. A presentation for $G_{i} /\left[G_{i}, G_{i}\right]$ is obtained by taking the $(|X|-1)\left[G: G_{i}\right]+1$ generators, then abelianising, and then quotienting by the $|R|\left[G: G_{i}\right]$ relations. Let $H_{i}$ be the abelian group obtained by the above procedure, but without quotienting by the relations that are lifts of $w^{q}$. This has $\mathbb{Z}^{\left[G: G_{i}\right]+1}$ as a summand.

We claim that $w \in G_{i}$ for each $i$. We will show this by induction. It clearly holds for $i=1$. Suppose therefore that $w \in G_{i}$. Now, the image of $w$ in $H_{i}$ lies in the subgroup of $H_{i}$ generated by $w^{q}$ and $w^{p_{i}}$, since $q$ is coprime to $p_{i}$. So, $w \in G_{i+1}$, proving the claim.

Suppose now that, for some $i, w$ has infinite order in $H_{i}$. Let $n$ be the largest integer (possibly zero) such that $w \in q^{n} H_{i}$. Set $K$ to be $\left[G_{i}, G_{i}\right]\left(G_{i}\right)^{q^{n+1}}$. Then $G / K$ is a finite homomorphic image of $G$ in which $w$ is non-trivial. A lemma of Baumslag and Pride in [2] then asserts that $K$, and hence $G$, is large. (Alternatively, one can avoid using this lemma of Baumslag and Pride, by showing directly that $K$ has a presentation with at least two more generators than relations, and then using Corollary 1.3.)

Hence, we may assume that $w$ has finite order in $H_{i}$ for each $i$. So, when we quotient $H_{i}$ by the relations that are lifts of $w^{q}$, a $\mathbb{Z}^{\left[G: G_{i}\right]+1}$ summand remains. Therefore, $G_{i} / G_{i+1}$ contains $\left(\mathbb{Z} / p_{i} \mathbb{Z}\right)^{\left[G: G_{i}\right]+1}$ as a summand. So,

$$
\begin{gathered}
d\left(G_{i} / G_{i+1}\right) \geqslant\left[G: G_{i}\right]+1, \\
\log \left[G_{i}: G_{i+1}\right] \geqslant \log \left(p_{i}{ }^{\left[G: G_{i}\right]+1}\right)=\left(\left[G: G_{i}\right]+1\right) \log p_{i} .
\end{gathered}
$$

Applying Theorem 1.1 then gives the corollary.

\section{Groups with positive virtual first Betti number}

In this final section, we establish Theorem 1.5. The implication $(1) \Rightarrow(2)$ is straightforward. For, if some finite index normal subgroup $G_{1}$ of $G$ has infinite abelianisation, we may then find finite index subgroups $G_{1} \geqslant G_{2} \geqslant \cdots$, each normal in $G_{1}$, so that $\left[G: G_{i}\right.$ ] grows as fast as we like. In particular, we may ensure that $\left[G_{i}: G_{i+1}\right]$ grows super-exponentially as a function of $\left[G: G_{i}\right]$.

The implication $(2) \Rightarrow(1)$ is a consequence of the following proposition.

Proposition 6.1. Let $G$ be a finitely presented group. Then, there is a constant $k$ with the following property. If $H$ is a finite index subgroup of $G$, then either its abelianisation $H / H^{\prime}$ is infinite or $\left|H / H^{\prime}\right| \leqslant k^{[G: H]}$.

Proof. Pick some finite presentation for $G$. Let $c$ denote the maximal length of any of its relations, and set $d$ to be the number of its generators. Let $K$ be the 2-complex arising from this presentation. Let $\widetilde{K} \rightarrow K$ be the cover corresponding to a finite index subgroup $H$. For $j=0,1,2$, let $C_{j}$ be the chain group generated by the $j$-cells of $\widetilde{K}$, and let $\partial_{2}: C_{2} \rightarrow C_{1}$ 
and $\partial_{1}: C_{1} \rightarrow C_{0}$ be the boundary maps. Then $H / H^{\prime}$ equals $\operatorname{Ker}\left(\partial_{1}\right) / \operatorname{Im}\left(\partial_{2}\right)$. Pick a maximal tree in the 1 -skeleton of $\widetilde{K}$. From this, one can construct a basis of $\operatorname{Ker}\left(\partial_{1}\right)$, where each element corresponds to an edge not in the tree, and is a loop formed by the edge and the path in the tree joining its endpoints. Thus, $\operatorname{Ker}\left(\partial_{1}\right)$ is a product of $[G: H](d-1)+1$ copies of $\mathbb{Z}$. Now, $\operatorname{Im}\left(\partial_{2}\right)$ is spanned by the images of the 2-cells. Each runs over at most $c$ 1-cells in $\widetilde{K}$, and so maps to a vector in $C_{1}$ with length at most $c$. We may suppose that $\left|H / H^{\prime}\right|$ is finite, and hence that the image of $\partial_{2}$ is a finite index subgroup of $\operatorname{Ker}\left(\partial_{1}\right)$. Pick a minimal collection $\mathcal{C}$ of 2 -cells such that $\partial_{2}(\langle\mathcal{C}\rangle)$ has finite index in $\operatorname{Ker}\left(\partial_{1}\right)$. Then, the images of these 2-cells span a parallelepiped in $\operatorname{Ker}\left(\partial_{1}\right)$. Denote its volume by $V$, which equals $\left|\operatorname{Ker}\left(\partial_{1}\right) / \partial_{2}(\langle\mathcal{C}\rangle)\right|$. But $\left|H / H^{\prime}\right|$ is a quotient of $\operatorname{Ker}\left(\partial_{1}\right) / \partial_{2}(\langle\mathcal{C}\rangle)$, and so $\left|H / H^{\prime}\right|$ is at most $V$. Since each 2 -cell in $\mathcal{C}$ maps to a vector in $\operatorname{Ker}\left(\partial_{1}\right)$ with length at most $c$, we deduce that the volume $V$ is at most $c^{[G: H](d-1)+1} \leqslant c^{d[G: H]}$. Thus, the proposition is proved by setting $k$ to $c^{d}$.

\section{Acknowledgments}

I am grateful to Peter Shalen for a discussion at the early stages of this project, when he suggested that a result along the lines of Theorem 1.1 might be true. I am also grateful to Alex Lubotzky for some useful conversations about Property $(\tau)$, when we discussed conditions similar to those in Theorem 1.1.

\section{References}

[1] B. Baumslag, S. Pride, Groups with two more generators than relators, J. London Math. Soc. 17 (2) (1978) 425-426.

[2] B. Baumslag, S. Pride, Groups with one more generator than relators, Math. Z. 167 (1979) 279-281.

[3] D. Gabai, Foliations and the topology of 3-manifolds, III, J. Differential Geom. 26 (1987) 479-536.

[4] M. Gromov, Volume and bounded cohomology, IHES Publ. Math. 56 (1982) 5-99.

[5] M. Lackenby, Expanders, rank and graphs of groups, Israel J. Math., in press.

[6] A. Lubotzky, Discrete groups, expanding graphs and invariant measures, Progr. Math. 125 (1994).

[7] A. Lubotzky, B. Weiss, Groups and expanders, in: Expanding Graphs, Princeton, 1992, in: DIMACS Ser. Discrete Math. Theoret. Comput. Sci., vol. 10, Amer. Math. Soc., Providence, RI, 1993, pp. 95-109.

[8] A. Lubotzky, R. Zimmer, Variants of Kazhdan's property for subgroups of semisimple groups, Israel J. Math. 66 (1989) 289-299.

[9] M. Scharlemann, Handbook of Knot Theory, Elsevier, in press.

[10] R. Stöhr, Groups with one more generators than relators, Math. Z. 182 (1983) 45-47. 